\title{
The new Heliospheric Magnetic Field: Observational Implications
}

\author{
T. H. Zurbuchen, L. A. Fisk, S. Hefti and N. A. Schwadron \\ Department of Atmospheric, Oceanic and Space Sciences, University of Michigan, Ann Arbor, USA
}

\begin{abstract}
A summary of the new model of the heliospheric magnetic field and its observational implications is presented. We first introduce a global model for the steady-state configuration in the low corona and discuss solar and heliospheric implications of the resulting field configuration. Finally, we compare the effects of this model with random transport of field-lines due to reconnection on the solar surface and to the dynamic turbulent transport of magnetic fieldlines.
\end{abstract}

\section{INTRODUCTION: THE THEORY}

One of the most unexpected discoveries of the Ulysses mission is the observation of recurrent events of lowrigidity particles observed up to very high latitude (1). These particles are accelerated at low latitude CoRotating Interaction Regions (CIRs) and propagate along heliospheric magnetic field-lines. However, if the heliospheric magnetic field has a Parker spiral configuration, the field-aligned latitude transport is not possible.

In his 1996 paper Fisk (2) described a model of the heliospheric magnetic field which has a substantially different geometry than the original Parker spiral configuration. The changes arise from the fact that solar magnetic field-lines expand from a differentially rotating photosphere into more rigidly rotating coronal holes which are not symmetrically centered on the solar rotation axis $\Omega$. The motions of the differentially rotating photospheric field-lines are then projected into the heliosphere, which results in deviations from the Parker spiral configuration.

It has been pointed out that the expansion of the solar magnetic field has to be considered as a global effect (3), (4). Close to the Sun plasma cannot support any net magnetic forces. The only alternative is then no pressure gradients in the magnetic field strength (5). Therefore, the magnetic field becomes uniform in strength in the corona, and thus creates a magnetic field in the heliosphere which has uniform strength $R^{2} B_{r}$, as is observed in situ (see, e.g. on Helios (6) and Ulysses (7)). Figure 1 illustrates a surface away from the Sun, where the constant pressure is established. The surface is placed at several solar radii from the Sun, but within the Alfven point. The surface is permeated only by open magnetic field-lines. It can be shown by Fisk (4) that the velocities occurring on this surface follow $\nabla \cdot \mathbf{u}_{\perp}=0$. All trajectories therefore

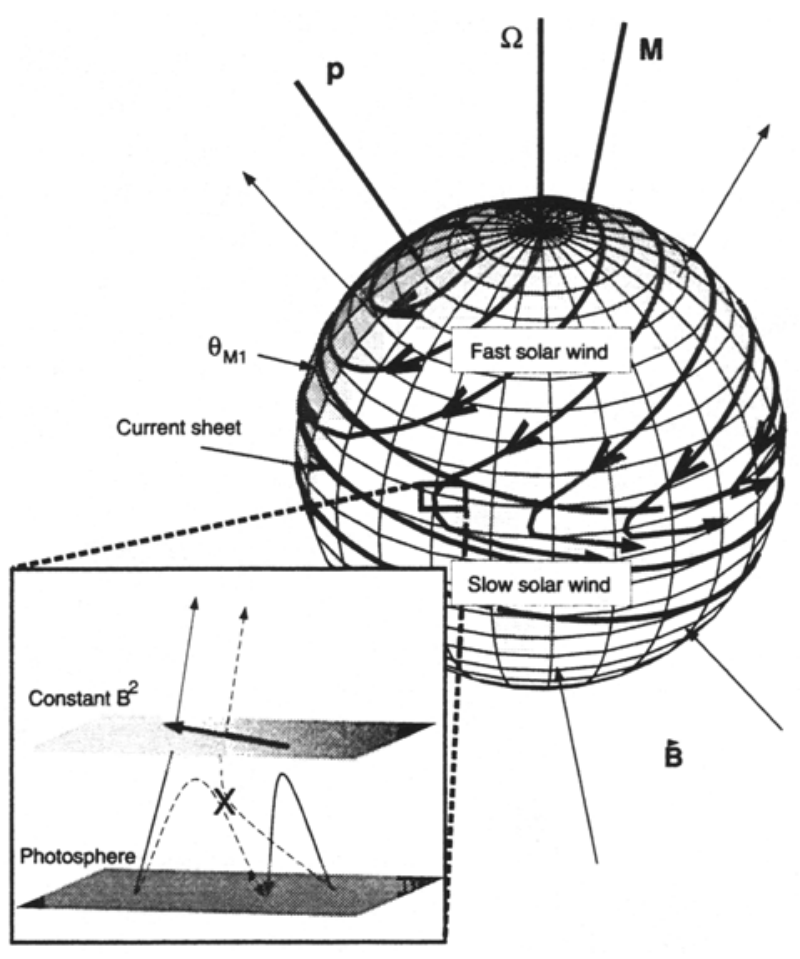

FIGURE 1. The surface of constant magnetic pressure. The surface is located at several solar radii from the Sun, and is penetrated only by open magnetic field-lines. The surface is located in a region of low beta plasma, and the field-lines are primarily radial. The location of the equatorial current sheet and the interface between the regions of fast and slow solar wind, $\theta_{M 1}$, are marked. The $M$-axis is normal to the plane defined by the equatorial current sheet, and is offset from the solar rotation axis $\Omega$. The field-line located at $p$ connects to the solar heliographic pole. The arrows on the surface are the trajectories of the fieldlines which penetrate through the surface, and are described in the text. 
have to be closed. There is an additional constraint on these trajectories: Polarities close to the Sun are strictly separated by the heliospheric current sheet and therefore $\mathbf{u}_{\perp}=\mathbf{0}$ through the current sheet. Because of this effect, the low latitude corona is intimately linked to high latitudes, where $u_{\perp}$ is determined by the super-expansion of high-latitude field-lines, and their differential rotation (8). At low latitudes, the transport mechanism must be qualitatively different from the situation at high latitudes, and the typical magnetic field topology is no longer open; reconnection has to occur. Failure to perform this reconnection would yield a very unnatural and insupportable bend in the field-line. The top end of the field-line must move to avoid magnetic pressure buildup. The bottom end must find a way to follow. As the field-line traverses back to the other side of the Sun at low latitudes, it presumably does so through a series of reconnections, which are illustrated schematically in the insert in Figure 1.

We will now discuss the implications of this magnetic field model for the large scale heliosphere, in particular at high latitude, and, for the nature of the solar wind. Finally, we will compare the effects of the differential rotation with other effects leading to considerable random latitude transport of magnetic field-lines.

\section{HIGH LATITUDE HELIOSPHERE}

In Figure 1, the magnetic field configuration at high latitudes is shown in its simplest form as would be expected close to activity minimum: The differential rotation at high latitude is mapped into closed curves about an axis $p$, the expanded magnetic field-line rooted in the heliographic pole of the photosphere. In this approximation, the magnetic field in the heliosphere is determined by two rotations (for details, see (2), and (8)). The first motion is given by the equatorial rotation period $\Omega$, equivalent to the Parker model. The Fisk model includes a second motion associated with the differential rotation of the photosphere on a time-scale of $\tau_{\text {diff }}=2 \pi /\left(\Omega-\Omega_{\text {pole }}\right) \sim 100$ days.

In spite of the simplicity of the foot-point motions at high latitudes, visualizing the three-dimensional geometry resulting from this model turns out to be rather challenging. We have attempted to visualize this in a set of movies which can be found on the CD-ROM accompanying these proceedings (refer to readme.note in the appropriate directory) or also on the world wide web on http://solar-heliospheric.engin.umich.edu. We have provided comparisons of field-lines without footpoint motion "Parker" and field-lines including footpoint motion "Fisk". For the simulations of the Fisk field we use the magnetic field model described in detail by Zurbuchen (8)

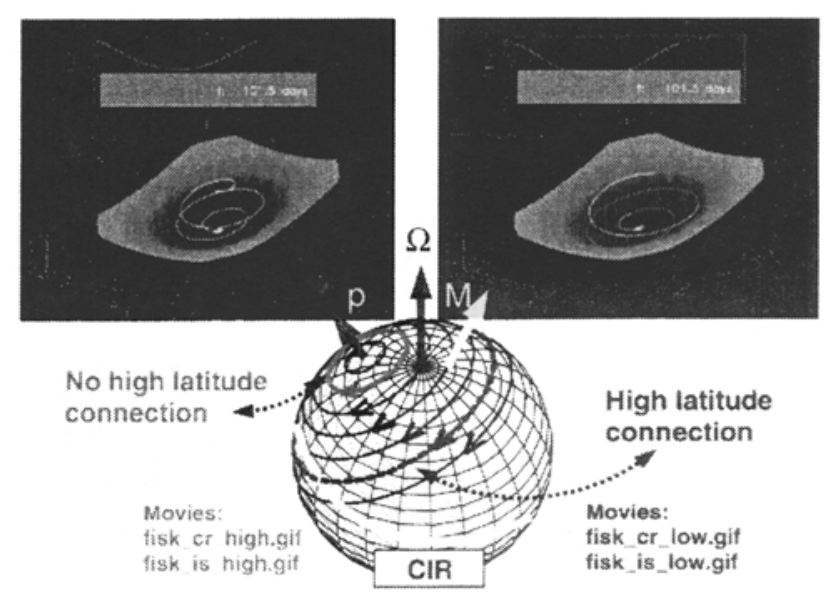

FIGURE 2. High latitude field connections in the outer heliosphere. The question whether a field-line connects to a CIR is determined by the trajectory of its footpoint on the surface of constant pressure and the three-dimensional geometry of the CIR which is assumed to be conical in this case. Field-lines from locations close to $p$ do not connect even though there latitude is not constant. Lower latitude field-lines do penetrate the CIR surface and can be loaded with low rigidity particles.

with a differential rotation time of $\tau_{\text {diff }}=100$ days and an offset angle between $p$ and $\Omega$ of $30^{\circ}$. The solar wind velocity is assumed to be radial with a constant magnitude of $800 \mathrm{~km} / \mathrm{sec}$. In order to discuss high-latitude connections we have assumed a conical CIR surface of a latitude of $30^{\circ}$. Realistically, this surface is of much more complicated geometry (9) but remains stationary in the frame co-rotating with the Sun. For each simulation we show a three-dimensional plot of the field-line and a radius-latitude plot of each point along the field-line. In the case of a Parker field-line we expect a straight line, since the latitude along the field-line does not change. We have used a field-line color scheme for the heliocentric distance; whenever the magnetic field-line is below the conical CIR surface, it has a red color.

We start out with field-lines in the frame co-rotating with the equatorial rotation speed of the Sun $\Omega$. At the simulation time $t=0$ the plasma leaving the Sun from one particular foot-point is marked with color. parker_cr_high.gif shows the time-history of the field-line in the 3D-heliosphere and a latitude-radius plot along the field-line. The latitude of the field-line is identically constant, as expected, and the slow solar wind blows the field out along the Parker spiral. For Fisk field-lines there are two different classes of high latitude field-lines as shown in Figure 2. The first one, shown in fisk_cr_high.gif is similar to the Parker field-line. It originates close to $p$ and even though it undergoes latitude variations, it does not connect with the CIR. Field-lines as shown in 
fisk_cr_low.gif allow for high-latitude connections to the CIR. Every single point of the Fisk field-lines is blown outwards by the solar wind as seen when concentrating on the first colored point of the field-lines. However, the shape of the field-line is changed by the additional slow rotation about $p$ including motions in the $\theta$ and $\phi$ direction. The former leads to high-latitude connections, the latter to under-and over-winding of the magnetic field.

In the inertial frame, the solar wind velocity is radial and the magnetic foot-points rotate about the Sun. This is shown for the Parker case in parker_is_high.gif. For the two cases of Fisk field-lines it is given by fisk_is_high.gif and fisk_is_low.gif. Notice two relevant aspects: First of all, the typical connection distance of field-lines from high-latitude to the CIR is of the order 10-20 AU. Secondly, there is a new class of field-lines in the outer heliosphere which are connected twice to the CIR forming huge arcs which connect back to the CIR on both sides.

This magnetic field configuration has important consequences for energetic particles in the outer heliosphere. Low energy particles propagating along the high latitude magnetic field will show the most significant effects as discussed by Fisk (1996) (2). As a first priority this field configuration should be included in a more realistic heliosphere model for example by Pizzo (9). There must be clear observational indications of this configuration, perhaps the arc-like structures discussed above for other particle populations observed for example on Voyager. Even though the drift pattern for very high energy particles does not change qualitatively, the field configuration should show quantitative effects.

\section{THE NATURE OF THE SOLAR WIND}

The global transport of the solar magnetic field on the surface of the Sun has important implications for the nature of the slow solar wind. We have stated before that the transport at high latitude is of different nature than the low-latitude transport where the coronal magnetic field is of typically closed topology and reconnection must occur. Remember, that this model for the corona, in which there are clear distinctions between the continuously open field-lines from the polar coronal hole, and randomly open field-lines at low latitude is not new but was proposed by Axford in (1977) (10). We should expect in these models, as is observed, that the slow wind is much more variable than the fast wind.

A direct consequence of this field configuration is an expected over- and under-winding of magnetic field at low latitude. As indicated in Figure 1, the magnetic flux has to return through the band of slow solar wind to the other side of the Sun. This return flux should result in

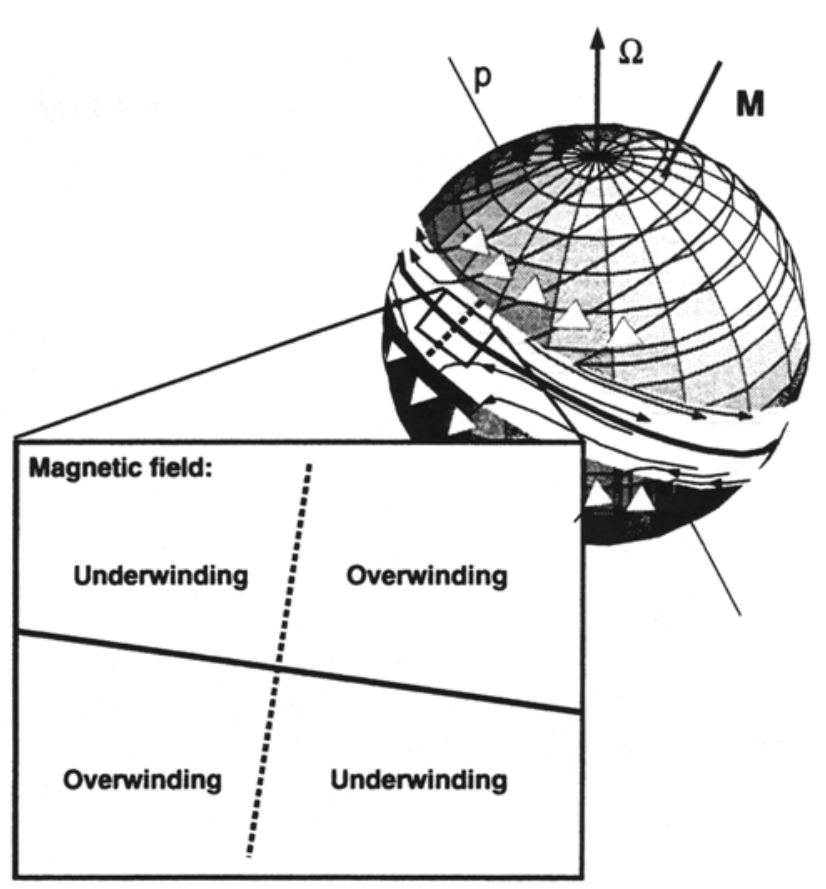

FIGURE 3. Predicted over- and under-winding at low latitudes. The low latitude magnetic field is predicted to show overwinding and under-winding depending on Carrington longitude and magnetic polarity.

over- or under-winding depending on Carrington longitude and magnetic polarity.

Recall that the slow wind is enhanced according the the first ionization potential (FIP) of the elements, whereas the fast wind is much less fractionated (11). This has been previously interpreted as a direct sign of a qualitative difference in fast and slow solar wind, consistent with the theory described here. It is possible, as done by Zurbuchen (12) and Schwadron (13), to construct a FIP enhancement mechanism which takes advantage of the fact that in this model the material which forms the slow solar wind started as material which was stored on closed loops.

Using high-resolution plasma and composition measurements such as the ion composition spectrometer SWICS on ACE we can also directly probe the transition between different wind streams as previously done with plasma data (14). It should be possible using composition data to check one relevant feature of this magnetic field theory, namely, whether there are indeed magnetic connections from fast to slow solar wind. This can be done best in the trailing edge of fast solar wind streams, which is dynamically spread out in time when observed on a spacecraft. If there are no magnetic connections from between fast and slow wind regions on the Sun, the transition would be expected to be step-like since mixing is 


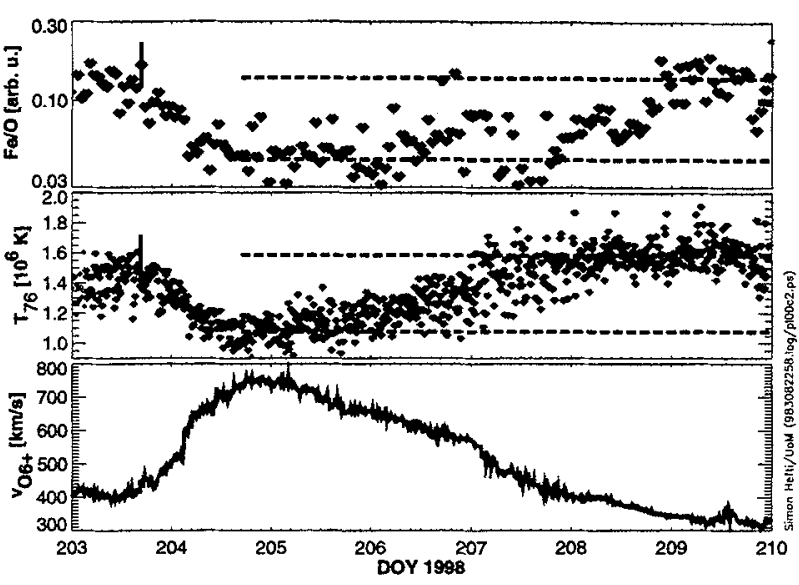

FIGURE 4. A transition from fast to slow wind from high timeresolution SWICS-ACE data. The Fe/O ratio is given in hourly averages, the freeze-in temperature $\mathrm{T}_{76}$ on a time-resolution of about 10 minutes. $\mathrm{T}_{76}$ is calculated from the ratio of the densities of $\mathrm{O}^{7+}$ and $\mathrm{O}^{6+}$. Also plotted is the Helium speed. The error-bars denote typical statistical uncertainties.

expected to be only effective along the magnetic field and very much suppressed in the perpendicular direction.

A first attempt has been undertaken by Zurbuchen (15), where the gradual transition of the composition in the trailing edge of a fast solar wind stream was interpreted as caused by a direct magnetic connection. Such a connection would enable mixing along field-lines resulting in a rather steady transition from "fast" to "slow" solar wind composition. Figure 4 shows an example of such a transition from fast to slow solar wind measured by SWICS-ACE (16). The elemental ratio of $\mathrm{Fe} / \mathrm{O}$ and the Oxygen freeze-in temperature $T_{76}$ are plotted during a five day transition from fast to slow solar wind. Clearly, a relatively steady transition from fast to slow solar wind composition is observed in elemental and charge composition. This measurement in Figure 4 is consistent with the existence of magnetic connections between fast and slow wind which would naturally lead to a steady transitions between "slow" and "fast" solar wind composition (15). However, this results needs to be critically examined and also put into the context of theoretical expansion profiles of the slow solar wind.

Using the same composition technique, it should be possible to analyze the "building blocks" of the slow solar wind by analyzing their compositional pattern and comparing them with solar remote sensing data (see discussion by Fisk (17)).

\section{RANDOM TRANSPORT OF MAGNETIC FIELD}

It has been argued that the observation of low energy particles at high latitude could result from a relatively large perpendicular diffusion of particles to high latitude (18). This diffusion could be caused by random magnetic reconnections in the photosphere or the low corona, leading to a braiding and twisting of field-lines on small spatial scales compared to a particle gyro-radius. This would allow particles to jump from field-line to field-line, therefore leading to a significant perpendicular diffusion, possibly larger than the effects from differential rotation of any one field-line. It has indeed been observed that the magnetic flux in the solar magnetic network reconfigures on a characteristic time-scale of $\tau \sim 1-2$ days (19). The characteristic scale size for the organization of magnetic field in the photosphere is the size of supergranules, or $\lambda \sim 30,000 \mathrm{~km}(19)$. The relatively random character of this reconnection process then results in a diffusion coefficient $\kappa=\lambda^{2} / \tau$.

It is now possible to compare the time-scales associated with this diffusive transport with the effective convective transport given by the differential rotation. The convection velocity on the solar surface is $v_{c}=\omega \cdot R_{\mathrm{s}}$, where $R_{\mathrm{s}}$ is a solar radius and $\omega=\Omega-\Omega_{\text {pole. }}$.

The time to cross a coronal hole of the size $\lambda_{c h} \sim 1 R_{S}$ is about $\tau_{\text {con }}=\lambda_{c h} / v_{c}$ using convection. When using the diffusive transport, it takes a typical time of $\tau_{\text {diff }}=\lambda_{\text {ch }}^{2} / \kappa$. With the given numbers the ratio of those time-scales is

$$
\tau_{\text {diff }} / \tau_{\text {con }} \sim 70 \text {. }
$$

This indicates that the effects of the differential rotation have to be clearly dominant over the effects of perpendicular diffusion caused by the magnetic reconnection described above.

A qualitatively very different perpendicular diffusion could result from the dynamical interaction of the solar wind in its dynamic and turbulent evolution (see, e.g. review articles by Goldstein et al. (20), and Tu and Marsch (21)). The turbulent mixing of a structure with the scale $\Lambda$ happens on a time-scale of $\tau_{\text {turb }}=\Lambda^{2} /\left(\Delta x^{2} / \Delta \tau\right)$, where $\Delta x^{2} / \Delta \tau$ stands for the diffusion coefficient of field-lines. This diffusion coefficient can be constrained by ACESWICS measurements with very high time-resolution (for instrumental details, see Gloeckler (16)). We determine the $\mathrm{O}^{7+} / \mathrm{O}^{6+}$ ratio during a time-period where the solar wind is essentially constant. As discussed previously, the low-speed solar wind has highly variable composition, which has clear variations down to 30 minutes. A very strong perpendicular diffusion coefficient would tend to smear out those gradients. The observation shows that $\tau_{\text {turb }}<r / V$, where $r=1 \mathrm{AU}$ and $V$ stands for the solar wind speed. This can be translated as a perpendicular 


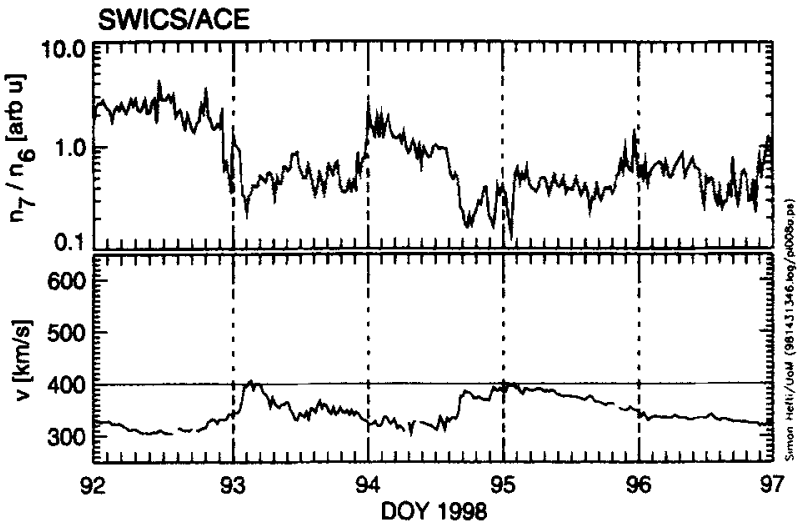

FIGURE 5. Solar composition measurements in low-speed solar wind. Even though the speed is more or less constant, the $\mathrm{O}^{7+} / \mathrm{O}^{6+}$ ratio has strong gradients on a measurement timescale of 30 minutes which are preserved from the low corona to $1 \mathrm{AU}$.

diffusion coefficient $\kappa_{\perp}$ for a particle of velocity $v$. Using $\Lambda \sim \Omega r \cdot 0.5 \mathrm{hrs}$, the limit on the turbulent diffusion turns out to be

$$
\kappa_{\perp}<v\left(3 \cdot 10^{8} \mathrm{~cm}\right) .
$$

Using a lower limit on the mean free path of $0.01 \mathrm{AU}$, $\kappa_{\perp} / \kappa_{\|}<0.002$. This is very small compared to values used before (18). This remains to be carefully investigated. It is conceivable that the composition boundaries are identical with tangential discontinuities which could be barriers to the turbulent evolution of the solar wind plasma. However, sharp transitions in the composition are very common and their number increases when going to increasingly better time-resolution (22). Even if the small diffusion coefficient only occur locally at discontinuities, there is a severe effect when averaging over large volumes in the heliosphere.

\section{DISCUSSION}

The inclusion of the differential rotation into the calculation of the expanding heliospheric magnetic field leads to interesting consequences in solar and heliospheric physics. There is already evidence for the existence for some of the predicted effects resulting from these consequences. However, more direct tests, some of which have been mentioned here, are possible and crucial.

Differential rotation effects make the solar magnetic field intrinsically time-dependent. Models with simplifying assumptions such as $B=\nabla \Phi$ will not be able to deal with this properly.

Additionally, the expansion of the solar magnetic field makes the large scale properties of the corona and coro- nal effects intrinsically three-dimensional. So far, simple analytic models have been used for these calculations. These models are highly constrained - for example, the dipole moment of the solar magnetic field is often not the dominant factor. However, the application of these theoretical concepts to more realistic solar magnetic field configurations is possible using modern numerical tools (23), (24).

\section{ACKNOWLEDGMENTS}

This work was supported, in part, by NASA contracts NAG5-2810, NAG5-7111 and JPL contract 955460. THZ and NAS were also supported, in part, by NASA grant NAG5-6471 and NSF grant ATM 9714070.

\section{REFERENCES}

1. Roelof, E. C., et al., J. Geophys. Res., 102, 251, (1997).

2. Fisk, L. A.,J. Geophys. Res., 101, 15547, (1996).

3. Fisk, L. A., Zurbuchen, T. H. and Schwadron, N. A., submitted to Astrophys. J.., (1999).

4. Fisk, L. A., Zurbuchen, T. H., and Schwadron, N. A., Space Sci. Rev., in press, (1999).

5. Suess, S. T., and Smith, E. J., Geophys. Res. Lett.,23, 3267, (1996).

6. Mariani, F., and Neubauer, F. M., in Physics of the inner heliosphere I, Springer Verlag, 183, (1990).

7. Balogh, A., E. J. Smith, et al., Science, 268, 1007, (1995).

8. Zurbuchen, T. H., Schwadron, N. A., and Fisk, L. A., J. Geophys. Res.,102, 24175, (1997).

9. Pizzo, V., J. Geophys. Res., 99, 4173, (1994).

10. Axford, W. I., in Study of Travelling Interplanetary Phenomena, eds M. A. Shea et al., Dordrecht: D. Reidel, 145, (1977).

11. Geiss, J., Gloeckler, G. and von Steiger, R., Space Sci. Rev., 72, 49, (1994).

12. Zurbuchen, T. H., Fisk, L. A., Gloeckler, G., and Schwadron, N. A., Space Sci. Rev., 85, 397, (1998).

13. Schwadron, N. A., Fisk, L. A. and Zurbuchen, T. H., submitted to Astrophys. J., (1998).

14. Schwenn, F. M., in Physics of the inner heliosphere I, Springer Verlag, 99, (1990).

15. Zurbuchen, T. H., Hefti, S., Fisk, L. A., Gloeckler, G. and von Steiger, R., Space Sci. Rev., in press, (1999).

16. Gloeckler, G. et al., Space Sci. Rev., 86, 495, (1998).

17. Fisk, L. A., Schwadron, N. A., and Zurbuchen, T. H., Space Sci. Rev., 96, (1998). 
18. Kota, J., and Jokipii, J. R., Science, 268, 1024, (1995).

19. Schrijver, et al., Astrophys. J., 487, 424, 1997.

20. Goldstein, M. L., Roberts, D. A. and Matthaeus, W. H., Ann. Rev. Astron. Astrophys., 33, 283, (1995).

21. Tu, C.-Y., and Marsch, E., Space Sci. Rev., 73, 1, (1995).

22. Hefti, S., PhD Thesis, University of Bern, 1997.

23. Groth, C. P. T., DeZeeuw, D. L., Gombosi, T. I., and Powell, K. G., Space Sci. Rev., in press, (1998).

24. Mikic, Z., and Linker, J. A., Astrophys. J., 430, 898, (1994). 